\title{
STABILIZATION OF THE WAVE EQUATION WITH MOVING BOUNDARY
}

\author{
KAÏS AMMARI, AHMED BCHATNIA, AND KARIM EL MUFTI
}

\begin{abstract}
We deal with the wave equation with assigned moving boundary $(0<x<a(t))$ upon which Dirichlet-Neuman boundary conditions are satisfied, here $a(t)$ is assumed to move slower than the light and periodically. We give a feedback which guarantees the exponential decay of the energy. The proof relies on a reduction theorem [1, 14. At the end we give a remark on the moving-pointwise stabilization problem.
\end{abstract}

\section{Contents}

1. Introduction and main result

2. Proof of the main result

3. Moving pointwise stabilization

References

\section{INTRODUCTION AND MAIN RESULT}

In this note, we analyze the stabilization property of solutions for the wave equation with a moving boundary. More precisely, we consider the following system:

$$
\left\{\begin{array}{l}
u_{t t}-u_{x x}=0 \text { for } 0<x<a(t), t>0 \\
u(0, t)=0 \text { and } u_{t}(a(t), t)+f(t) u_{x}(a(t), t)=0, t>0 \\
u(x, 0)=\phi(x), u_{t}(x, 0)=\psi(x), 0<x<a(0)
\end{array}\right.
$$

$(\phi, \psi) \in H_{l}^{1}((0, a(0))) \times L^{2}((0, a(0)))$, where

$$
H_{l}^{1}((0, a(0)))=\left\{v \in H^{1}((0, a(0))), v(0)=0\right\} .
$$

Here $a$ is a strictly positive real function which is continuous, 1-periodic and $f \in$ $L^{\infty}\left(\mathbb{R}_{+}^{*}\right)$.

Denote by

$$
E_{u}(t)=\frac{1}{2} \int_{0}^{a(t)}\left[\left|u_{t}(x, t)\right|^{2}+\left|u_{x}(x, t)\right|^{2}\right] d x
$$

the energy of the field $u$. Our major concern will be to detect the feedback $f(t)$ necessary to obtain the exponential decay of $E_{u}(t)$.

We start with some notations and known results. Let $\operatorname{Lip}(\mathbb{R})$ be the space of Lipschitz continuous functions on $\mathbb{R}$. We shall denote the Lipschitz constant of a function $F$ by

$$
L(F):=\sup _{x, y \in \mathbb{R}, x \neq y}\left|\frac{F(x)-F(y)}{x-y}\right| .
$$

On the existence of solutions to the system (1.1), we refer the reader to 9 . We have the following proposition:

2010 Mathematics Subject Classification. 35L05, 34K35, 93B07, 95B05.

Key words and phrases. Strings with moving ends, stabilization, rotation number. 
Proposition 1.1. If $a \in \operatorname{Lip}(\mathbb{R}), L(a) \in[0,1), a>0, f \in L^{\infty}\left(\mathbb{R}_{+}^{*}\right)$ and $(\phi, \psi) \in H_{l}^{1}((0, a(0))) \times L^{2}((0, a(0)))$, denote by $Q:=(0, a(t)) \times \mathbb{R}_{+}$and $Q_{\tau}:=(0, a(t)) \times(0, \tau), \tau \in \mathbb{R}_{+}$. There exists a unique weak solution $u$ of the system (1.1). Moreover there exists $h \in H_{\text {loc }}^{1}(\mathbb{R}) \cap L^{\infty}(\mathbb{R})$ such that

$$
u(x, t)=h(t+x)-h(t-x) \text { a.e. in } Q,
$$

and $u \in L^{\infty}(Q) \cap H^{1}\left(Q_{\tau}\right)$.

We denote by $D_{p}$ the set of continuous functions and strictly increasing of the form $x+g(x)$, where $g(x)$ is a 1-periodic continuous function.

We recall the following results.

Proposition 1.2. ([10, Herman] and [12, Yamaguchi]) Let a be a 1-periodic function. Then

$$
F:=(I+a) \circ(I-a)^{-1}
$$

belongs to $D_{p}$. Moreover, the rotation number $\rho(F)$ defined by

$$
\rho(F)=\lim _{n \rightarrow \infty} \frac{F^{n}(x)-x}{n}
$$

exists, and the limit is equal for all $x \in \mathbb{R}$.

After, we construct a transformation of the time-dependent domain $[0, a(t)] \times \mathbb{R}$ onto $[0, \rho(F) / 2] \times \mathbb{R}$ that preserves the D'Alembertian form of the wave equation. For this purpose, we use the following proposition:

Proposition 1.3. ([10, Herman, section II]) Assume that a $(t)$ is a 1-periodic function, $a(t)>0, a \in \operatorname{Lip}(\mathbb{R})$ such that $L(a) \in[0,1)$. Assume also that $\left|a^{\prime}(t)\right|<1$ for all $t \in \mathbb{R}$ and $\rho(F) \in \mathbb{R} \backslash \mathbb{Q}$ such that there exists a function $H \in D_{p}$ and

$$
H^{-1} \circ F \circ H(\xi)=\xi+\rho(F) .
$$

Our main result is stated now as follows:

Theorem 1.4 (Exponential stability). Let

$$
f(t)=\frac{(\mu-1) H^{\prime}(a(t)+t)+(\mu+1) H^{\prime}(-a(t)+t)}{(1-\mu) H^{\prime}(a(t)+t)+(\mu+1) H^{\prime}(-a(t)+t)}
$$

where $\mu$ is a nonnegative constant and assume that there exist $\lambda_{1}>0$ and $\lambda_{2}>0$ such that

$$
\lambda_{1} \leq H^{\prime}(t) \leq \lambda_{2}, t \in \mathbb{R} .
$$

Then, in the case where $\mu \neq 1$, there exists a positive constant $C$ such that

$$
E_{u}(t) \leq C e^{-\omega t} E_{u}(0)
$$

for every solution $u$ of (1.1) with initial data $(\phi, \psi) \in H_{l}^{1}((0, a(0))) \times L^{2}((0, a(0)))$ and where $\omega=\ln \left(\left|\frac{1+\mu}{1-\mu}\right|\right)$.

In the case $\mu=1$ which corresponds to $f(t)=1$, we obtain

$$
E_{u}(t)=0, \text { for all } t \geq T_{0}=:(I+a)^{-1} \circ H^{-1}\left(\frac{3 \rho(F)}{2}\right) .
$$

We give an example where assumption (1.6) is guaranteed (see [1, 8] for more details).

\footnotetext{
${ }^{1} u \in H^{1}\left(Q_{\tau}\right)$ is called a weak solution of (1.1) if $u_{t t}-u_{x x}=0$ in $\mathcal{D}^{\prime}(Q)$ and the boundary conditions are satisfied.
} 
Example 1.5. Let a be continuous and 1-periodic on $\mathbb{R}, a>0$, be such that

$$
a(t):= \begin{cases}\alpha t+\frac{\alpha(1-\alpha)(1+\beta)}{2(\alpha-\beta)} & \text { if } \frac{\alpha(1+\beta)}{2(\alpha-\beta)} \leq t \leq \frac{\alpha(1+\beta)-2 \beta}{2(\alpha-\beta)}, \\ \beta t-\beta+\frac{\alpha\left(1-\beta^{2}\right)}{2(\alpha-\beta)} & \text { if } \frac{\alpha(1+\beta)-2 \beta}{2(\alpha-\beta)} \leq t \leq \frac{\alpha(3+\beta)-2 \beta}{2(\alpha-\beta)},\end{cases}
$$

with $-1<\beta<0<\alpha<1$. The function $F$ is defined by:

$$
F(x):=(I+a) \circ(I-a)^{-1}(x)= \begin{cases}l_{1} x+F_{0} & \text { if } 0 \leq x \leq x_{0} \\ l_{2} x+F_{0}+1-l_{2} & \text { if } x_{0}<x<1\end{cases}
$$

with $l_{1}:=\frac{1+\alpha}{1-\alpha}, l_{2}:=\frac{1+\beta}{1-\beta}, F_{0}:=\frac{l_{2}\left(l_{1}-1\right)}{l_{1}-l_{2}}$ and $x_{0}:=\frac{1-l_{2}}{l_{1}-l_{2}}$.

We extend $F$ through the formula: $F(x+1)=F(x)+1$ for any $x \in \mathbb{R}$. Also the rotation number is given by the expression:

$$
\rho(F)=\frac{\ln l_{1}}{\ln \left(\frac{l_{1}}{l_{2}}\right)}
$$

and the function $H$ given by 1.4) is done by

$$
H(x)=h_{0} \ln \left(\left|x+h_{1}\right|\right)+h_{2},
$$

where $h_{0}=\frac{1}{\ln \left(\frac{l_{1}}{l_{2}}\right)}, h_{1}=\frac{l_{2}}{l_{1}-l_{2}}$ and $h_{2}=-\ln \left(h_{1}\right)$. H satisfies the inequalities:

$$
\frac{1}{\ln \left(\frac{l_{1}}{l_{2}}\right)} \frac{l_{1}-l_{2}}{l_{1}} \leq H^{\prime}(x) \leq \frac{1}{\ln \left(\frac{l_{1}}{l_{2}}\right)} \frac{l_{1}-l_{2}}{l_{2}} .
$$

Here $f(t)=\frac{2 a(t)+2 \mu t+2 \mu h_{1}}{2 \mu a(t)+2 t+2 h_{1}}$ and $T_{0}=(I+a)^{-1} \circ H^{-1}\left(\frac{3 \rho(F)}{2}\right)$.

Remark 1.6. For the moving pointwise stabilization of the wave equation, see section 3 .

\section{Proof of the MAIN RESUlt}

Before starting the proof, we begin by defining a domain transformation

$$
\Phi: \mathbb{R}^{2} \rightarrow \mathbb{R}^{2}
$$

using $H$ given by (1.4), as follows:

$$
\left\{\begin{array}{l}
\xi=(H(x+t)-H(-x+t)) / 2 \\
\tau=(H(x+t)+H(-x+t)) / 2
\end{array}\right.
$$

for $(x, t) \in \mathbb{R}^{2}$.

Proposition 2.1. ([13, Yamaguchi]) The transformation $\Phi$ is a bijection of $[0, a(t)] \times$ $\mathbb{R}$ to $[0, \rho(F) / 2] \times \mathbb{R}$ and $\Phi$ maps the boundaries $x=0$ and $x=a(t)$ onto the boundaries $\xi=0$ and $\xi=\rho(F) / 2$.

Proposition 2.2. ([13, Yamaguchi]) Let $u(x, t)$ satisfying $\left(\partial_{t}^{2}-\partial_{x}^{2}\right) u(x, t)=0$ and $V(\xi, \tau)$ defined by $u\left(\Phi^{-1}(\xi, \tau)\right)$. Then the following identity holds

$$
\left(\partial_{t}^{2}-\partial_{x}^{2}\right) u(x, t)=K(\xi, \tau)\left(\partial_{\tau}^{2}-\partial_{\xi}^{2}\right) V(\xi, \tau)
$$

where $K(\xi, \tau)$ is defined by

$$
4 H^{\prime} \circ H^{-1}(\xi+\tau) H^{\prime} \circ H^{-1}(-\xi+\tau) \circ H^{-1}(\xi+\tau) .
$$

Now we consider the system:

$$
\left\{\begin{array}{l}
u_{t t}-u_{x x}=0 \text { for } 0<x<a(t), t>0 \\
u(0, t)=0 \text { and } u_{t}(a(t), t)+f(t) u_{x}(a(t), t)=0, t>0 \\
u(x, 0)=\phi_{1}(x), u_{t}(x, 0)=\psi_{1}(x), \quad 0<x<a(0)
\end{array}\right.
$$


where $f(t)=\frac{(\mu-1) H^{\prime}(a(t)+t)+(\mu+1) H^{\prime}(-a(t)+t)}{(1-\mu) H^{\prime}(a(t)+t)+(\mu+1) H^{\prime}(-a(t)+t)}$ and $\mu$ is a nonnegative constant.

Proposition 2.3. The transformation of the system (2.13) is

$$
\left\{\begin{array}{l}
v_{\tau \tau}-v_{\xi \xi}=0 \quad \text { for } 0<\xi<\rho(F) / 2, \tau>0 \\
v(0, \tau)=0 \text { and } v_{\tau}(\rho(F) / 2, \tau)+\mu v_{\xi}(\rho(F) / 2, \tau)=0, \tau>0 \\
\left.v(\xi, 0)=\phi(\xi), v_{\tau}(\xi, 0)=\psi(\xi), \quad 0<\xi<\rho(F) / 2\right)
\end{array}\right.
$$

Proof. We have:

$$
\left\{\begin{array}{l}
u_{x}(a(t), t)=V_{\xi}(\rho(F) / 2, \tau) \xi_{x}(a(t), t)+V_{\tau}(\rho(F) / 2, \tau) \tau_{x}(a(t), t) \\
u_{t}(a(t), t)=V_{\xi}(\rho(F) / 2, \tau) \xi_{t}(a(t), t)+V_{\tau}(\rho(F) / 2, \tau) \tau_{t}(a(t), t)
\end{array}\right.
$$

Starting from (2.13) and make use of:

$$
\begin{aligned}
& \xi_{x}=\left(\partial_{x} \xi\right)=\left(\partial_{t} \tau\right)=\tau_{t}=\left[H^{\prime}(x+t)+H^{\prime}(-x+t)\right] / 2, \\
& \xi_{t}=\left(\partial_{t} \xi\right)=\left(\partial_{x} \tau\right)=\tau_{x}=\left[H^{\prime}(x+t)-H^{\prime}(-x+t)\right] / 2,
\end{aligned}
$$

we conclude:

$$
u_{t}(a(t), t)+\frac{\xi_{t}-\mu \tau_{t}}{\mu \tau_{x}-\xi_{x}} u_{x}(a(t), t)=0
$$

So we have

$$
\xi_{t} u_{x}(a(t), t)-\xi_{x} u_{t}(a(t), t)+\mu\left(\tau_{x} u_{t}(a(t), t)-\tau_{t} u_{x}(a(t), t)\right)=0 .
$$

Finally we get: $v_{\tau}(\rho(F) / 2, \tau)+\mu v_{\xi}(\rho(F) / 2, \tau)=0$.

Note that $f(t)=1$ in the special case $\mu=1$.

The next lemma shows that the energy of the solution (1.1) and the energy of the corresponding static system are equivalent.

Lemma 2.4. ([1, Ammari et al.]) Under the assumption (1.6), there are two positive constants $C_{1}$ and $C_{2}$ such that

$$
C_{1} E_{V}\left(H(a(t)+t)-\frac{\rho(F)}{2}\right) \leq E_{u}(t) \leq C_{2} E_{V}\left(H(a(t)+t)-\frac{\rho(F)}{2}\right),
$$

where $E_{V}(\tau)$ is the energy of the field $V$ defined by:

$$
E_{V}(\tau)=\int_{0}^{\rho(F) / 2}\left(\left|V_{\xi}(\xi, \tau)\right|^{2}+\left|V_{\tau}(\xi, \tau)\right|^{2}\right) d \xi .
$$

Note that we can write $H(a(t)+t)=a(t)+t+g(a(t)+t)$, where $g(x)$ is a 1-periodic continuous function.

Finally, the stabilization of the system (1.1) is a direct combination of Proposition 2.3. Lemma 2.4 and the following Lemma 2.5 .

Lemma 2.5. ([7, Cox and Zuazua]) For $\mu \neq 1$, there exists a positive constant $C$ such that

$$
E_{V}(\tau) \leq C e^{-\ln \left(\left|\frac{1+\mu}{1-\mu}\right|\right) \tau} E_{V}(0), \forall \tau>0,
$$

where $V$ is the solution of the following system:

$$
\left\{\begin{array}{l}
V_{\tau \tau}-V_{\xi \xi}=0 \text { for } 0<\tau<\rho(F) / 2, \tau>0, \\
V(0, \tau)=0 \text { and } V_{\tau}(\rho(F) / 2, \tau)+\mu V_{\xi}(\rho(F) / 2, \tau)=0, \tau>0 \\
\left.V(\xi, 0)=\phi(\xi), V_{\tau}(\xi, 0)=\psi(\xi), \quad 0<\xi<\rho(F) / 2\right) .
\end{array}\right.
$$

It is well known that for $\mu=1, E_{V}(t)=0$ for all $t \geq \rho(F)$, see [7] for more details. 


\section{MOVInG POINTWise STABILIZATION}

We consider the following problem:

$$
\left\{\begin{array}{l}
u_{t t}-u_{x x}+\left[f_{1}(t) u_{t}+f_{2}(t) u_{x}\right] \delta_{a(t)}=0 \text { for } 0<x<b(t), t>0 \\
u(0, t)=0 \text { and } u(b(t), t)=0, t>0 \\
u(x, 0)=\phi_{1}(x), u_{t}(x, 0)=\psi_{1}(x) 0<x<b(0) .
\end{array}\right.
$$

The aim of this section is to determine the functions $f_{1}, f_{2}$ and $b$ to get after transformation the vibrations of a string with the static pointwise damping and conclude the asymptotic behavior of the energy.

Proposition 3.1. The transformation of the system:

$$
\left\{\begin{array}{l}
u_{t t}-u_{x x}+K\left(\frac{H(a(t)+t)-H(-a(t)+t)}{2}, \frac{H(a(t)+t)+H(-a(t)+t)}{2}\right) \\
\cdot\left[\left(\frac{1}{H^{\prime}(a(t)+t)}-\frac{1}{H^{\prime}(-a(t)+t)}\right) u_{t}+\left(\frac{1}{H^{\prime}(a(t)+t)}+\frac{1}{H^{\prime}(-a(t)+t)}\right) u_{x}\right] \delta_{a(t)}=0 \\
\quad f o r \quad 0<x<b(t)=\Lambda_{t}^{-1}(1)-t, t>0 \\
u(0, t)=0 \text { and } u(b(t), t)=0, t>0 \\
u(x, 0)=\phi_{1}(x), u_{t}(x, 0)=\psi_{1}(x), 0<x<b(0)
\end{array}\right.
$$

$i s$

$$
\left\{\begin{array}{l}
v_{\tau \tau}-v_{\xi \xi}+v_{\tau} \delta_{\frac{\rho(F)}{2}}=0 \quad \text { for } \quad 0<\xi<1, \tau>0 \\
v(0, \tau)=0 \text { and } v(1, \tau)=0, \tau>0 \\
v(\xi, 0)=\phi(\xi), v_{\tau}(\xi, 0)=\psi(\xi), \quad 0<\xi<1
\end{array}\right.
$$

where $\Lambda_{t}$ is defined by $\Lambda_{t}(y)=\frac{H(y+t)-H(-y+t)}{2}$.

Proof. We recall that if $u(x, t)$ satisfying $\left(\partial_{t}^{2}-\partial_{x}^{2}\right) u(x, t)=0$ and $v(\xi, \tau)$ defined by $u\left(\Phi^{-1}(\xi, \tau)\right)$. Then the following identity holds

$$
\left(\partial_{t}^{2}-\partial_{x}^{2}\right) u(x, t)=K(\xi, \tau)\left(\partial_{\tau}^{2}-\partial_{\xi}^{2}\right) v(\xi, \tau)
$$

where $K(\xi, \tau)$ is defined by

$$
4 H^{\prime} \circ H^{-1}(\xi+\tau) H^{\prime} \circ H^{-1}(-\xi+\tau) \circ H^{-1}(\xi+\tau) .
$$

On the other hand, make use of (2.15) we obtain:

$$
\begin{aligned}
v_{\tau} & =\frac{\xi_{t} u_{x}-\xi_{x} u_{t}}{\xi_{t}^{2}-\xi_{x}^{2}} \\
& =\left(\frac{1}{H^{\prime}(a(t)+t)}-\frac{1}{H^{\prime}(-a(t)+t)}\right) u_{t}+\left(\frac{1}{H^{\prime}(a(t)+t)}+\frac{1}{H^{\prime}(-a(t)+t)}\right) u_{x} .
\end{aligned}
$$

We combine (3.22) and (3.23) to obtain the result of the Proposition 3.1.

Remark 3.2. If we return to the Example 1.5 and after some computation we get that the transformation of the system:

$$
\left\{\begin{array}{l}
u_{t t}-u_{x x}+\left(\frac{8}{a(t)+t+h_{1}} \cdot \frac{a(t)+t}{-a(t)+t+h_{1}}\right) \cdot\left[a(t) u_{t}+\left(t+h_{1}\right) u_{x}\right] \delta_{a(t)}=0 \\
f o r \quad 0<x<b(t)=\left(t+h_{1}\right) e^{\frac{h_{2}}{h_{0}}} \tanh \left(\frac{1}{h_{0}}\right), t>0 \\
u(0, t)=0 \text { and } u(b(t), t)=0, t>0 \\
u(x, 0)=\phi_{1}(x), u_{t}(x, 0)=\psi_{1}(x), 0<x<b(0)
\end{array}\right.
$$

is the system (3.21).

As above and according to [4, 5] we have the following:

- $\lim _{t \rightarrow+\infty} E_{u}(t)=0, \forall\left(\phi_{1}, \psi_{1}\right) \in H_{0}^{1}(0, b(0)) \times L^{2}(0, b(0)) \Leftrightarrow \rho(F) \notin \mathbb{Q}$.

- For any $\rho(F) / 2 \in(0,1)$ the system (3.20) is not exponentially stable in $H_{0}^{1}(0, b(0)) \times L^{2}(0, b(0))$. 
- For all $\rho(F) / 2 \in \mathcal{S} 2$ and for all $\left(\phi_{1}, \psi_{1}\right) \in \mathcal{D}$ we have according to [2, 3] that there exists $C>0$ such that:

$$
E_{u}(t) \leq \frac{C}{t}, \forall t>0
$$

- If $\varepsilon>0$ then, for almost all $\rho(F) / 2 \in(0,1)$ and for all $\left(\phi_{1}, \psi_{1}\right) \in \mathcal{D}$ we have according to [2, 3] that there exists $C>0$ such that:

$$
E_{u}(t) \leq \frac{C}{t^{\frac{1}{1+\varepsilon}}}, \forall t>0
$$

where

$$
\begin{gathered}
\mathcal{D}:=\left\{(\varphi, \psi) \in\left[H^{2}(0, b(0)) \cap H_{0}^{1}(0, b(0))\right] \times H_{0}^{1}(0, b(0))\right. \\
\left.\left(\frac{1}{H^{\prime}(a(0))}-\frac{1}{H^{\prime}(-a(0))}\right) \psi(a(0))+\left(\frac{1}{H^{\prime}(a(0))}+\frac{1}{H^{\prime}(-a(0))}\right) \frac{d \varphi}{d x}(a(0))=0\right\} .
\end{gathered}
$$

\section{REFERENCES}

[1] K. Ammari, A. Bchatnia and K. El Mufti, A remark on observability of the wave equation with moving boundary, Journal of Applied Analysis, in press, 1 (2017).

[2] K. Ammari and M. Tucsnak, Stabilization of second order evolution equations by a class of unbounded feedbacks, ESAIM Control Optim. Calc. Var., 6 (2001), 361-386.

[3] K. Ammari and S. Nicaise, Stabilization of elastic systems by collocated feedback, Lecture Notes in Mathematics, 2124. Springer, Cham, 2015.

[4] K. Ammari, A. Henrot and M. Tucsnak, Asymptotic behaviour of the solutions and optimal location of the actuator for the pointwise stabilization of a string, Asymptot. Anal., 28 (2001), 215-240.

[5] Optimal location of the actuator for the pointwise stabilization of a string, C. R. Acad. Sci. Paris Sr. I Math., 330 (2000), 275-280.

[6] J. W. S. Cassals, An introduction to Diophantine Approximation, Cambridge University Press, Cambridge (1966).

[7] S. Cox and E. Zuazua, The Rate at which energy decays in a string damped at one end, Comm. Partial Differential Equations., 19 (1994), 213-243.

[8] N. Gonzalez, An example of pure stability for the wave equation with moving boundary, $J$. Math. Anal. Appl., 228 (1998), 51-59.

[9] L L'équation des ondes dans un domaine dépendant du temps, Ph.D. Thesis, University of Toulon and Czech Technical University, 1997.

[10] M. Herman, Sur la conjugaison différentiable des difféomorphismes du cercle à des rotations, Publ. Math. I.H.E.S., 49 (1979), 5-234.

[11] S. Lang, Introduction to diophantine approximations, Addison Wesley, New York (1966).

[12] M. Yamaguchi, Periodic solutions of nonlinear equations of string with periodically oscillating boundaries, Funkcialaj. Ekvacioj., 45 (2002), 397-416.

[13] M. Yamaguchi and H. Yoshida, Nonhomogeneous string problem with periodically moving boundaries, Fields Inst. Commun., 25 (2000), 565-574.

[14] J. C. Yoccoz, Conjugaison différentiable des difféomorphismes du cercle dont le nombre de rotation vérifie une condition diophantienne, Ann. Sci. École Norm. Sup., 17 (1984), 333359.

UR Analysis and Control of PDEs, UR13ES64, Department of Mathematics, Faculty of Sciences of Monastir, University of Monastir, 5019 Monastir, Tunisia

E-mail address: kais.ammari@fsm.rnu.tn

UR Analyse Non-Linéaire et Géométrie, UR13ES32, Department of Mathematics, FaCUlty of Sciences of Tunis, University of Tunis El Manar, Tunisia

E-mail address: ahmed.bchatnia@fst.utm.tn

UR Analysis and Control of PDEs, UR13ES64, ISCAE, University of Manouba, Tunisia E-mail address: karim.elmufti@iscae.rnu.tn

\footnotetext{
${ }^{2}$ Denote by $\mathcal{S}$ the set of all numbers $\xi \in(0,1)$ such that $\xi \notin \mathbb{Q}$ and if $\left[0, a_{1}, \ldots, a_{n}, \ldots\right]$ is the expansion of $\xi$ as a continued fraction, then $\left(a_{n}\right)$ is bounded. Let us notice that $\mathcal{S}$ is is obviously uncountable and, by classical results on diophantine approximation (cf. [6], p. 120), its Lebesgue measure is equal to zero. In particular, by EulerLagrange theorem (see Lang [11, p. 57) S contains all $\xi \in(0,1)$ such that $\xi$ is an irrational quadratic number (i.e. satisfying a second degree equation with rational coefficients).
} 